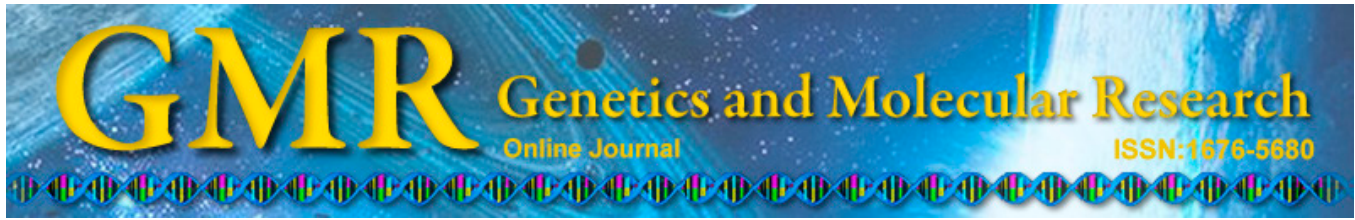

\title{
Well-balanced commensal microbiota contributes to anti-cancer response in a lung cancer mouse model
}

\author{
Q.-F. Gui ${ }^{1}$, H.-F. Lu ${ }^{2}$, C.-X. Zhang ${ }^{1}$, Z.-R. Xu' ${ }^{1}$ and Y.-M. Yang ${ }^{1}$ \\ ${ }^{1}$ Department of Geriatrics, First Affiliated Hospital, School of Medicine, \\ Zhejiang University, Hangzhou, Zhejiang, China \\ ${ }^{2}$ Department of Infectious Disease, First Affiliated Hospital, \\ School of Medicine, Zhejiang University, Hangzhou, Zhejiang, China \\ Corresponding author: Y.-M. Yang \\ E-mail: yyuhai@163.com
}

Genet. Mol. Res. 14 (2): 5642-5651 (2015)

Received August 18, 2014

Accepted December 8, 2014

Published May 25, 2015

DOI http://dx.doi.org/10.4238/2015.May.25.16

\begin{abstract}
The intestinal microflora affects inflammation and immunity, not only locally at the mucosal level but also systemically, raising the question of whether the microflora affects inflammatory processes that contribute to cancer and its therapy. Prebiotics have also been found to play an antitumor role that is not limited to the gut. We investigated the antitumor roles of the intestinal microbiota using the Lewis lung cancer mouse model. In mice treated with cisplatin combined with ABX (an antibiotic cocktail of vancomycin, ampicillin, and neomycin), which can destroy the host commensal microflora, the tumor size was larger than in mice on a single treatment of cisplatin. Moreover, the survival rate of mice treated with cisplatin combined with $\mathrm{ABX}$ was significantly reduced. In contrast, mice treated with cisplatin combined with Lactobacillus bacteria had smaller tumors and an improved survival rate. Further study on gene expression indicated that $\mathrm{ABX}$ can partially impair the function of cisplatin by upregulating the expression of VEGFA and downregulating the expression of BAX
\end{abstract}


and CDKN1B. The expression of IFN- $\gamma$, GZMB, and PRF1 in the CD8 ${ }^{+}$ $\mathrm{T}$ cells of these mice was reduced by $\mathrm{ABX}$, indicating an immunoenhancement role of commensal microbiota. Conversely, Lactobacillus co-treatment mice showed an enhanced antitumor response with upregulated IFN- $\gamma$, GZMB, and PRF1 expression. We conclude that the commensal microbiota contributes to the anti-lung cancer response and probiotics co-treatment can enhance the antigrowth and proapoptotic effects of cisplatin.

Key words: Lung cancer; Lactobacillus; Cisplatin; Immune response

\section{INTRODUCTION}

Lung cancer is one of the most common causes of cancer death and has a high mortality rate in both men and women worldwide. At present, platinum is one of the most widely used regimens for the treatment of lung cancer. Although more than $80 \%$ of patients with lung cancer are responsive to chemotherapy, they are seldom cured by it because of rapidly developed drug resistance. The 5-year survival rate of lung cancer patients is less than 20\% (Miller, 2005). Therefore, developing new clinical diagnosis and treatment methods is extremely important.

The commensal microbiota of the gut influences metabolism, tissue development, inflammation, and immunity (Lee and Mazmanian, 2010). Increasingly, research indicates that the intestinal microflora plays an important role in some kinds of cancer pathogenesis. Previously, the intestinal microflora has been found to produce reactive species such as the derivatives of molecular oxygen, including superoxide, hydrogen peroxide, and hydroxyl radicals, which contribute to genomic instability and are related to the initiation of various kinds of cancer (Lax, 2005; Waris and Ahsan, 2006). However, until recently, the anticancer protection function of well-balanced intestinal microflora has only partially been unveiled (Iida et al., 2013).

According to the World Health Organization's 2001 definition, probiotics are "live microorganisms, which, when administered in adequate amounts, confer a health benefit on the host". Probiotics in the intestine mainly refer to the genera Lactobacillus and Bifidobacterium. Many studies have outlined the beneficial effects of probiotics on various aliments such as food allergies (Kalliomäki and Isolauri, 2003), inflammatory bowel disease, and ulcerative colitis (Stephani et al., 2011). Probiotics exert their modulatory actions by different mechanisms, including: anti-genotoxicity; inhibition of colonic enzyme activity, which reduces the harmful effects of carcinogens activated by enzymatic action in the colon (Liong, 2008); control of the growth of potentially harmful bacteria (Zarei et al., 2009); interaction with colonocytes; and enhancement of homeostasis and the integrity of the epithelial barrier. Recently, research by Hijová et al. (2012) on colon cancer rats has indicated that prebiotics have a protective effect and may be useful for colon cancer prevention and treatment. Furthermore, the protective effect from prebiotics is not only limited to the digestive system. Research by Maroof et al. (2012) group has shown that Lactobacillus acidophilus enhances the immune response against breast cancer in a murine model. In the current study, to understand whether the intestinal microflora exerts anti-lung cancer effects, we fed mice with an antibiotic cocktail of vancomycin, ampicillin, and neomycin (ABX) to destroy their intestinal microflora balance. To investigate the protection function of intestinal prebiotics, an independent group of mice were fed with Lactobacillus bacteria. We examined tumor 
size, mouse survival rate, expression of two oncogenes and two cancer suppressor genes, and serum cytokine levels to determine the function of the intestinal microbiota in a lung cancer mouse model.

\section{MATERIAL AND METHODS}

\section{Cells and reagents}

Lewis lung cancer (LLC) cells were purchased from the Shanghai Institute of Cell Biology (Shanghai, China), maintained in Dulbecco's modified Eagle's medium (Gibco, Carlsbad, CA, USA) supplemented with $10 \%$ fetal bovine serum (Gibco), $100 \mathrm{U} / \mathrm{mL}$ penicillin, and $100 \mu \mathrm{g} / \mathrm{mL}$ streptomycin, and incubated at $37^{\circ} \mathrm{C}$ in a humidified atmosphere containing $5 \% \mathrm{CO}_{2}$. Cisplatin was purchased from Sigma Aldrich Chemical Co. (St. Louis, MO, USA). Vancomycin, imipenem, and neomycin were purchased from North China Pharmaceutical Group Corp (Shijiazhuang, Hebei, China).

\section{Lactobacillus bacteria}

A Lactobacillus acidophilus strain was used in this study as a probiotic and was purchased from the Institute of Microbiology Chinese Academy of Science. Before administration, the L. acidophilus strain was grown aerobically overnight at $37^{\circ} \mathrm{C}$ in de Man, Rogosa, and Sharpe broth (Oxoid). The following day, bacteria were harvested by centrifugation, washed twice with sterile phosphate-buffered saline (PBS), and adjusted to a cell density of 2 x $10^{8}$ colony-forming units (CFU) per milliliter.

\section{In vivo tumor growth assay}

Seventy-two C57BL/6J male mice (6-8 weeks old) were purchased from the Shanghai Laboratory Animal Center (Shanghai, China). Mice were given free access to pellet food and water, and were kept in plastic cages at $21^{\circ} \pm 2^{\circ} \mathrm{C}$ on a 12 -h light-dark cycle. All animals received humane care. The animal protocol was reviewed and approved by the Animal Care and Research Committee of Zhejiang University. LLC cells were cultured in 100-mm dishes and collected in $15-\mathrm{mL}$ centrifuge tubes. The cells were then centrifuged $(800 \mathrm{~g}, 5 \mathrm{~min})$ and washed twice with ice-cold PBS. The cells were diluted to $1 \times 10^{6}$ cells $/ \mathrm{mL}$ and injected subcutaneously into the right flanks of the male $\mathrm{C} 57 \mathrm{BL} / 6 \mathrm{~J}$ mice. The mice were randomly divided into four groups. All mice formed tumors 3 days after injection. Five days after injection, each group of mice was subjected to a different treatment: 1) cisplatin group: intraperitoneal injection of cisplatin (5 mg per kg body weight) combined with feeding PBS; 2) cisplatin/ABX group: intraperitoneal injection of cisplatin ( $5 \mathrm{mg}$ per kg body weight) combined with feeding an ABX antibiotic cocktail; 3) cisplatin/L. acidophilus: intraperitoneal injection of cisplatin (5 mg per kg body weight) combined with feeding an L. acidophilus solution containing $2 \times 10^{8}$ $\mathrm{CFU} / \mathrm{mL}$ via the orogastric route; and 4) control: intraperitoneal injection of PBS combined with feeding PBS. Tumor sizes were monitored every 5 days and volume was calculated using the following formula:

Volume $\left(\mathrm{mm}^{3}\right)=($ longest diameter $) \times(\text { shortest diameter })^{2} / 2$ 
Mice were euthanized when they became moribund.

\section{Western blotting}

Protein extracts from cells were boiled in sodium dodecyl sulfate/ $\beta$-mercaptoethanol sample buffer, and $20 \mu \mathrm{g}$ samples were loaded into each $10 \%$ polyacrylamide gel lane. The proteins were separated by electrophoresis, and the proteins in the gels were blotted onto polyvinylidene difluoride membranes (Amersham Pharmacia Biotech, St. Albans, Herts, UK) by electrophoretic transfer. The membranes were incubated with rabbit anti-VEGFA, -BAX, and -CDKN1B polyclonal antibodies (Abcam, Cambridge, MA, USA) or Ras monoclonal antibody separately for $2 \mathrm{~h}$ at $37^{\circ} \mathrm{C}$. The specific protein-antibody complex was detected using horseradish peroxidase-conjugated goat anti-rabbit or rabbit anti-mouse IgG. Detection by the chemiluminescence reaction was carried using the ECL kit (Pierce; Appleton, WI, USA). The $\beta$-actin signal was used as a loading control. The band intensity was analyzed by using the Quantity One software (Bio-Rad).

\section{RNA extraction and quantitative reverse transcription-polymerase chain reaction (qRT-PCR) analysis}

Total RNA was extracted from tumor samples using TRIzol reagent (Invitrogen, Carlsbad, CA, USA) according to manufacturer instructions. Briefly, about $100 \mathrm{mg}$ tissue sample was homogenized using TRIzol reagent, and was incubated at room temperature for 5 min. Chloroform was added to the samples, vigorously mixed, and incubated at room temperature for $5 \mathrm{~min}$. Following incubation, the samples were centrifuged at 12,000 $\mathrm{g}$ for $15 \mathrm{~min}$ at $4^{\circ} \mathrm{C}$. RNA was precipitated from the aqueous phase by addition of isopropyl alcohol to a fresh tube containing the supernatant aqueous phase. The integrity of the RNA was tested by spectroscopic analysis.

For the reverse transcription reaction, $1 \mu \mathrm{g}$ extracted RNA was used for reverse transcription with Oligo(dT) primers. For qRT-PCR analysis, $1 \mu \mathrm{g}$ cDNA products was used, and the primers were as follows: Ifn- $\gamma$ sense (5'-3'): ATGAACGCTACACACTGCATC, antisense (5'-3'): CCATCCTTTTGCCAGTTCCTC; $P r f 1$ sense (5'-3'): AGCACAAGTTCGTGCCAGG, anti-sense (5'-3'): GCGTCTCTCATTAGGGAGTTTTT; Gzmb sense (5'-3'): CCACTCTCGA CCCTACATGG, anti-sense (5'-3'): GGCCCCCAAAGTGACATTTATT; $R N 18 S$ sense (5'-3'): AGTCCCTGCCCTTTGTACACA, anti-sense (5'-3'): CGATCCGAGGGCCTCACTA.

\section{Serum levels of interferon (IFN)- $\gamma$, interlukin (IL)-6, IL-10, and tumor necrosis factor (TNF)- $\alpha$ detection}

Serum samples from mice were used for the measurement of serum levels IFN- $\gamma$, IL6 , IL-10, and TNF- $\alpha$ by the enzyme-linked immunosorbent assay (ELISA) kit (R\&D Systems, Minneapolis, MN, USA), according to manufacturer instructions.

\section{Preparation of mouse $\mathrm{CD8}^{+} \mathrm{T}$ cells}

Mouse $\mathrm{CD} 8^{+} \mathrm{T}$ cells were isolated from the lymph nodes with a Dynabeads FlowComp Mouse CD8 Kit (Invitrogen) according to manufacturer protocols. Briefly, $1 \mathrm{~mL}$ (5 
x $10^{7}$ cells) isolated from the lymph nodes were transferred to a tube and incubated with 50 $\mu \mathrm{L}$ FlowComp Mouse CD8 antibody for $10 \mathrm{~min}$ at $4^{\circ} \mathrm{C}$. The cells were resuspended in $2 \mathrm{~mL}$ isolation buffer $\left(\mathrm{Ca}^{2+}\right.$ - and $\mathrm{Mg}^{2+}$-free PBS supplemented with $0.1 \%$ bovine serum albumin and $2 \mathrm{mM}$ ethylenediaminetetraacetic acid after being washed once with $4 \mathrm{~mL}$ isolation buffer. Then, the suspensions were incubated with $150 \mu \mathrm{L}$ washed FlowComp Dynabeads for 15 $\min$ at $4^{\circ} \mathrm{C}$ while rolling and tilting. Then, $2 \mathrm{~mL}$ isolation buffer was added, and the tube was placed in a magnet for $2 \mathrm{~min}$. The supernatant containing CD8 - cells was carefully removed and discarded, while the tube was still in the magnet. The bead-bound $\mathrm{CD} 8^{+}$cells were washed once with isolation buffer, and the $\mathrm{CD} 8^{+}$cells were released from the beads. The total RNA was extracted using TRIzol reagent.

\section{Statistical analysis}

All the data were analyzed using the Student $t$-test with the SPSS 17.0 software package. The overall survival curves were plotted according to the Kaplan-Meier method, and the generalized log-rank test was applied to compare the survival curves. The findings were considered to be significant at a $\mathrm{P}<0.05$.

\section{RESULTS}

The commensal microflora influences the metabolism, tissue development, and immunity of the human body. Recently, increasing evidence indicates that the intestinal microflora plays important roles in the process of the anti-cancer response. Moreover, the effects do not only take place in the digestive system. To investigate the protection function of commensal microflora against lung cancer, we established an LLC-bearing tumor model in C57BL/6J mice. As expected, the tumor size was significantly reduced in the cisplatin-treated mice $(\mathrm{P}<$ 0.01) (Figure 1A).

However, when the tumor-bearing mice were treated with cisplatin combined with $\mathrm{ABX}$, which destroys the commensal microflora homeostasis, the tumor size was slightly reduced by cisplatin, although the difference was not significant $(\mathrm{P}>0.05)$. The results indicate that well-balanced commensal microflora homeostasis is helpful for the anti-tumor response. Moreover, when the mice were treated with cisplatin and fed Lactobacillus bacteria via the orogastric route, the tumor size was reduced significantly compared with mice that were treated with only cisplatin and PBS. These phenomena hint that the intestinal microflora participates in the body's anti-lung cancer response, and the antitumor function of prebiotics can reach the lung.

To confirm the above results further, we examined the survival rate of the lung cancerbearing mice on the different treatments. As shown in Figure 1B, compared with cisplatin treatment alone, cisplatin combined with $\mathrm{ABX}$-treated mice had shorter $(\mathrm{P}=0.011)$ survival times and the cisplatin combined with $L$. acidophilus-treated mice had longer survival times $(\mathrm{P}=0.048)$.

To reveal the antitumor function of commensal microflora and prebiotics, we detected the expression of two oncogenes (Vegfa and Ras) and two tumor suppressor genes (Cdknlb and $B a x$ ) in the tumor by western blotting. As shown in Figure 2, the expression levels of VEGFA and RAS were reduced to 56.13 and $67.22 \%$ by cisplatin, respectively. 
A

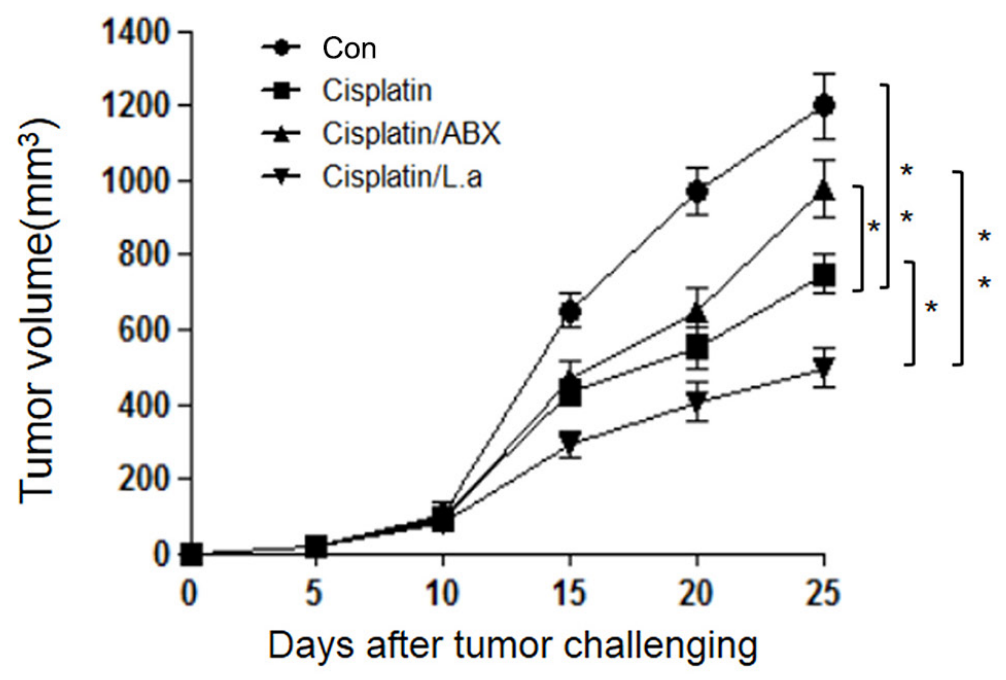

B

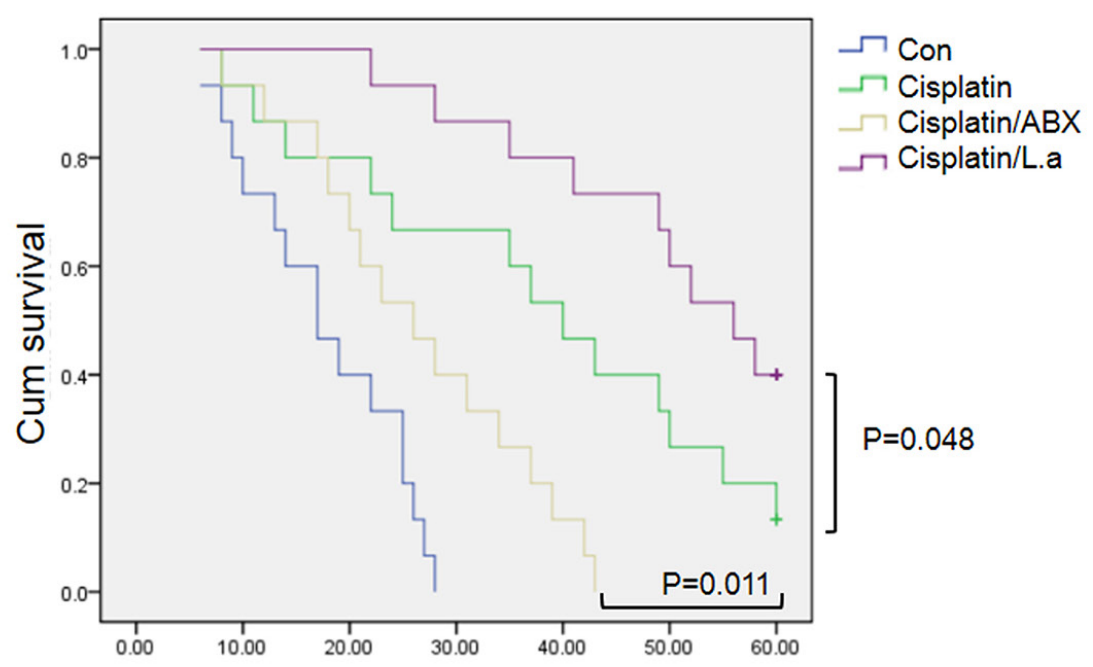

Days after tumor challenging

Figure 1. Commensal microbiota contributes to anti-cancer response in Lewis lung cancer (LLC)-bearing mice. Mice were divided into 4 groups: Cisplatin group, $5 \mathrm{mg} / \mathrm{kg}$ cisplatin combined with feeding phosphatebuffered saline (PBS); Cisplatin/ABX group, $5 \mathrm{mg} / \mathrm{kg}$ cisplatin combined with ABX feeding (antibiotic cocktail of vancomycin, ampicillin, and neomycin); Cisplatin/L.a group, $5 \mathrm{mg} / \mathrm{kg}$ cisplatin combined with feeding with Lactobacillus acidophilus (L.a.) solution containing $2 \times 10^{8} \mathrm{CFU} / \mathrm{mL}$ via the orogastric route; and control (Con) group, intraperitoneal injection of PBS combined with feeding PBS. The results were analyzed by the Student $t$-test (A). Tumor sizes were monitored every 5 days; the overall survival curves were plotted according to the KaplanMeier method (B). ${ }^{*} \mathrm{P}<0.05,{ }^{*} \mathrm{P}<0.01$. 
A

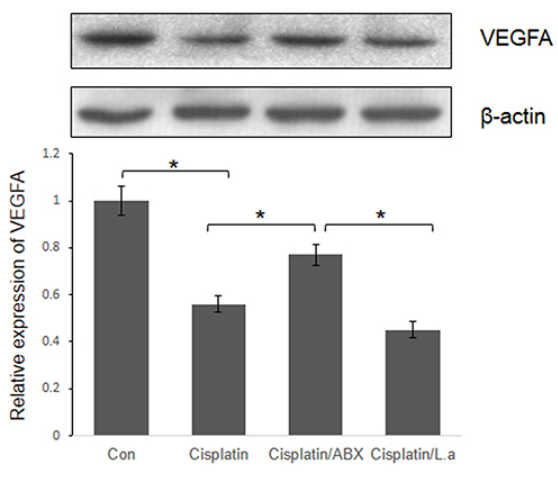

C

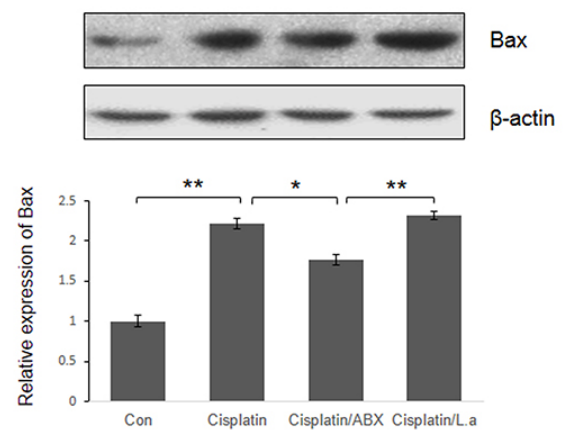

B

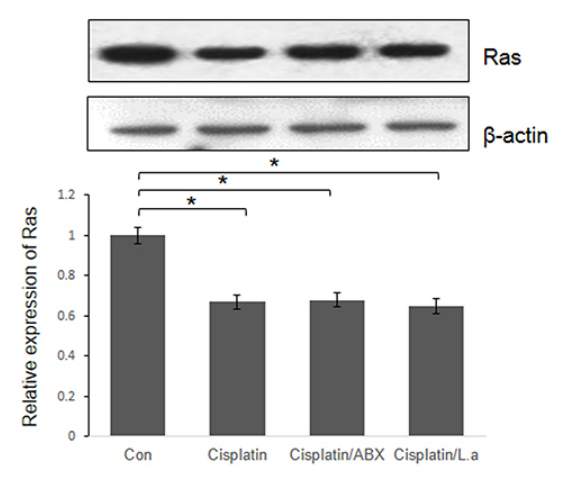

D

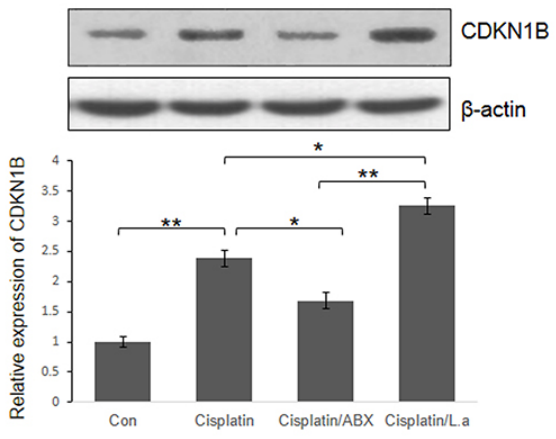

Figure 2. Antibiotics and Lactobacillus bacteria change the antigrowth and proapoptotic effects of cisplatin on Lewis lung cancer (LLC) xenografts. At the 25th day after tumor challenging, three mice from each group were sacrificed and the expression levels of VEGFA (A), Ras (B), Bax (C), and CDKN1B (D) were detected by western blot. The $\beta$-actin signal was used as a loading control. The band intensities were analyzed using the Quantity One software and the results were analyzed by the Student $t$-test. $* \mathrm{P}<0.05, * * \mathrm{P}<0.01$. ABX is an antibiotic cocktail of vancomycin, ampicillin, and neomycin; L.a. is Lactobacillus acidophilus.

The expression levels of BAX and CDKN1B were upregulated to 120.88 and $138.24 \%$, respectively. Furthermore, treatment with $\mathrm{ABX}$ partially impaired the influence of cisplatin on the expression of VEGFA, BAX, and CDKN1B. In contrast, Lactobacillus bacteria enhanced the effect of cisplatin, especially with regards to the expression of VEGFA and CDKN1B.

We next used ELISA to detect the levels of four serum cytokines in the mice on the 25 th day after tumor challenging. As shown in Figure 3A, the levels of the four cytokines were not significantly changed in the mice by treatment with cisplatin alone compared with the control mice.

The serum IFN- $\gamma$ level was reduced by $28.7 \%$ when the mice were treated with cisplatin combined with $\mathrm{ABX}$ compared with treatment by cisplatin alone. When treated with the cisplatin/L. acidophilus combination, the serum IFN- $\gamma$ level was upregulated by $44.1 \%$ compared with cisplatin alone. Furthermore, the serum IL-6 level in the cisplatin/L. acidophi- 
lus group was 1.89-fold that of the cisplatin/ABX group, and the IL-10 level of cisplatin/ $L$. acidophilus mice was only $51.2 \%$ that of the cisplatin/ABX mice.

A
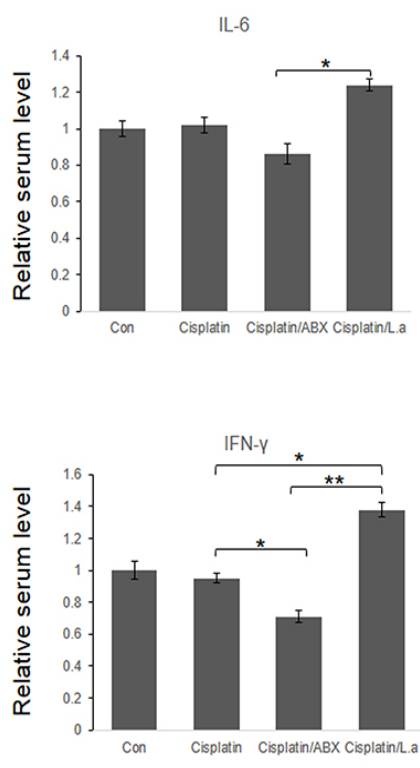

B

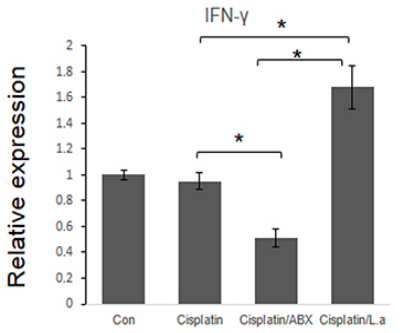

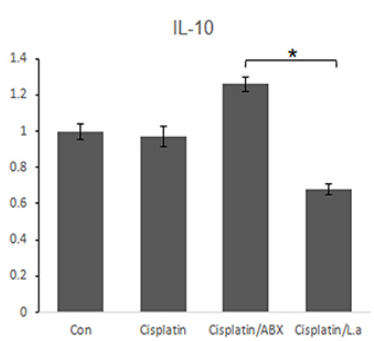

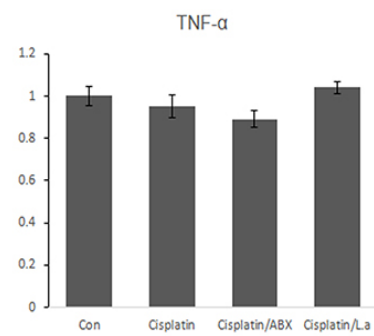

Figure 3. Serum cytokine levels and king ability of $\mathrm{CD} 8^{+} \mathrm{T}$ cells were altered by antibiotics and Lactobacillus bacteria. A. At the 25th day after tumor challenging, three mice from each group were sacrificed and the serum levels of IFN- $\gamma$, IL-6, IL-10, and TNF- $\alpha$ were detected by enzyme-linked immunosorbent assay (ELISA). B. $\mathrm{CD}^{+}$cells were isolated from the lymph nodes and the mRNA levels of Ifn- $\gamma, G z m b$, and Prflwere detected by quantitative reverse transcription polymerase chain reaction (qRT-PCR). Results were analyzed by the Student $t$-test and $\mathrm{P}<0.01$ was considered to be statistically significant. $* \mathrm{P}<0.05, * * \mathrm{P}<0.01$. ABX is an antibiotic cocktail of vancomycin, ampicillin, and neomycin; L.a. is Lactobacillus acidophilus.

Finally, we isolated the $\mathrm{CD} 8^{+} \mathrm{T}$ cells from the mouse lymph nodes and the mRNA levels of Ifn- $\gamma, P r f 1$, and Gzmb were detected by real-time qRT-PCR (Figure 3B). The results showed that cisplatin treatment did not change the expression of these three genes. However, ABX treatment reduced the mRNA levels by $49.1 \%$ (Ifn- $\gamma), 34.8 \%$ (Gzmb), and $40.6 \%$ (Prfl) compared with the mice treated with cisplatin alone. Furthermore, L. acidophilus treatment upregulated the mRNA levels by $66.7 \%(I f n-\gamma), 56.8 \%(G z m b)$, and $118.9 \%(P r f l)$ compared with the mice treated with cisplatin alone. 


\section{DISCUSSION}

The intestinal microflora affects inflammation and immunity, not only locally at the mucosal level but also systemically, raising the question of whether the microflora affects inflammatory processes that contribute to cancer and its therapy. Prebiotics have also been found to play an antitumor role that is not limited to the gut. We investigated the antitumor roles of the intestinal microbiota using the LLC mouse model, and report our finding here. In mice treated with cisplatin combined with $\mathrm{ABX}$, which can destroy host commensal microflora, the tumor size was larger than in mice on a single treatment of cisplatin. Moreover, the survival rate of mice treated with cisplatin combined with $\mathrm{ABX}$ was significantly reduced. In contrast, mice treated with cisplatin combined with Lactobacillus bacteria had smaller tumors and an improved survival rate. Further study on gene and protein expression indicated that ABX can partially impair the function of cisplatin by upregulating the expression of VEGFA and downregulating the expression of BAX and CDKN1B. The expression of IFN- $\gamma$, GZMB, and PRF1 in the $\mathrm{CD} 8^{+} \mathrm{T}$ cells of these mice was reduced by $\mathrm{ABX}$, indicating an immuno-enhancement role of commensal microbiota. Conversely, Lactobacillus co-treatment mice showed an enhanced antitumor response with upregulated Ifn- $\gamma, G z m b$, and PrfI mRNA expression.

VEGFA is involved in both vasculogenesis and angiogenesis, and overexpression of VEGF is observed in lung cancer patients. CDKN1B is considered a tumor suppressor because of its function as a regulator of the cell cycle. BAX is known to function as a promoter of apoptosis by binding to and antagonizing BCL-2. In this study, the tumors of the cisplatin/ABX group mice had upregulated VEGFA expression and downregulated expression of CDKN1B and BAX, indicating increased malignancy and reduced cisplatin function. Conversely, the tumors of the cisplatin/L. acidophilus group mice had reduced malignancy, which means that the Lactobacillus bacteria contributed to the antitumor response. Furthermore, ABX inhibited the expression of IFN- $\gamma$, GZMB, and PRF1 in the CD8 ${ }^{+}$T cells. In contrast, L. acidophilus upregulated the expression of IFN- $\gamma$, GZMB, and PRF1, and thereby facilitates the antigrowth and proapoptotic effects of cisplatin.

In conclusion, the results of this study collectively suggest that the commensal microbiota contributes to the anti-lung cancer response, and probiotics co-treatment can enhance the antigrowth and proapoptotic effects of cisplatin.

\section{REFERENCES}

Hijová E, Bomba A, Bertková I, Strojný L, et al. (2012). Prebiotics and bioactive natural substances induce changes of composition and metabolic activities of the colonic microflora in cancerous rats. Acta Biochim. Pol. 59: 271-274.

Iida N, Dzutsev A, Stewart CA, Smith L, et al. (2013). Commensal bacteria control cancer response to therapy by modulating the tumor microenvironment. Science 342: 967-970.

Kalliomäki M and Isolauri E (2003). Role of intestinal flora in the development of allergy. Curr. Opin. Allergy Clin. Immunol. 3: 15-20.

Lax AJ (2005). Opinion: Bacterial toxins and cancer - a case to answer? Nat. Rev. Microbiol. 3: 343-349.

Lee YK and Mazmanian SK (2010). Has the microbiota played a critical role in the evolution of the adaptive immune system? Science 330: 1768-1773.

Liong MT (2008). Roles of probiotics and prebiotics in colon cancer prevention: Postulated mechanisms and in-vivo evidence. Int. J. Mol. Sci. 9: 854-863.

Maroof H, Hassan ZM, Mobarez AM and Mohamadabadi MA (2012). Lactobacillus acidophilus could modulate the immune response against breast cancer in murine model. J. Clin. Immunol. 32: 1353-1359.

Miller YE (2005). Pathogenesis of lung cancer: 100 year report. Am. J. Respir. Cell. Mol. Biol. 33: 216-223. 
Stephani J, Radulovic K and Niess JH (2011). Gut microbiota, probiotics and inflammatory bowel disease. Arch. Immunol. Ther. Exp. 59: 161-177.

Waris G and Ahsan H (2006). Reactive oxygen species: role in the development of cancer and various chronic conditions. J. Carcinog. 5: 14.

Zarei S, Schwenter F, Luy P, Aurrand-Lions M, et al. (2009). Role of GM-CSF signaling in cell-based tumor immunization. Blood 113: 6658-6668. 